\title{
HEPATOLOGY
}

\section{Phenotyping non-alcoholic fatty liver disease by the gut microbiota: Ready for prime time?}

\author{
Münevver Demir, ${ }^{*}$ Sonja Lang, ${ }^{\dagger, \ddagger}$ Anna Martin, ${ }^{\dagger}$ (D) Fedja Farowski, ${ }^{\S, \eta, * *}$ Hilmar Wisplinghoff, ${ }^{\dagger+, \neq \neq, \S \S}$

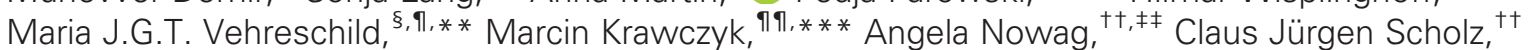 \\ Anne Kretzschmar, ${ }^{+\dagger}$ Christoph Roderburg, ${ }^{*}$ Frank Lammert, ${ }^{\text {甲ा }}$ Tobias Goeser, $^{\dagger}$ Philipp Kasper $^{\dagger}$ and \\ Hans-Michael Steffen ${ }^{\dagger}$
}

* Department of Hepatology and Gastroenterology, Campus Virchow Clinic and Campus Charité Mitte, Charité University Medicine, Berlin, Germany ${ }^{\dagger}$ University of Cologne, Faculty of Medicine, and University Hospital Cologne, Department of Gastroenterology and Hepatology, Cologne, Germany ${ }^{\ddagger}$ Department of Medicine, University of California San Diego, La Jolla, California USA ${ }^{\S}$ University of Cologne, Faculty of Medicine, and University Hospital Cologne, Department I of Internal Medicine, Cologne, Germany "German Centre for Infection Research (DZIF), partner site Bonn/ Cologne ${ }^{* *}$ Department of Internal Medicine, Infectious Diseases, Goethe University Frankfurt, Frankfurt am Main, Germany ${ }^{\dagger \dagger}$ Wisplinghoff Laboratories, Cologne, Germany ${ }^{\ddagger}$ University of Cologne, Faculty of Medicine, Institute for Medical Microbiology, Immunology and Hygiene, University Hospital of Cologne, Cologne, Germany ${ }^{\S \S}$ Institute for Virology and Medical Microbiology, University Witten/Herdecke, Witten, Germany ${ }^{\text {I1 }}$ Department of Medicine II, Saarland University Medical Center, Saarland University, Homburg, Germany*** Laboratory of Metabolic Liver Diseases, Department of General, Transplant and Liver Surgery, Medical University of Warsaw, Warsaw, Poland

\section{Key words}

dysbiosis, microbiome, NAFL, NASH.

Accepted for publication 3 April 2020.

\section{Correspondence}

Münevver Demir, Department of Hepatology and Gastroenterology, Campus Virchow Clinic and Campus Charité Mitte, Charité University Medicine. Augustenburger Platz 1, Berlin DE13353, Germany.

Email: muenevver.demir@charite.de

Declaration of conflict of interest: No conflicting financial interests to declare.

\begin{abstract}
Background and Aim: Several studies observed alterations in the gut microbiota in patients with non-alcoholic fatty liver disease (NAFLD). However, analyzed patient populations and methods strongly differ among these studies. The aim of this study was to prove the reproducibility of published results and to provide a detailed overview of all findings in our NAFLD cohort using next generation sequencing methods.

Methods: The individual taxonomic microbiota composition of fecal samples from 90 NAFLD patients and 21 healthy controls was analyzed using 16S rRNA gene sequencing. Study participants were grouped according to their disease stage and compared regarding their gut microbiota composition. Studies were identified from PubMed listed publications, and the results were compared with the findings in our cohort.

Results: Results from 13 identified studies were compared with our data. A decreased abundance of the Bacteroidetes and Ruminococcaceae as well as an increased abundance of Lactobacillaceae and Veillonellaceae and Dorea were the most frequently reported changes among NAFLD patients in 4/13, 5/13, 4/13, 2/13, and 3/13 studies, respectively. Even though these alterations in the gut microbiota composition were also observed in our patient cohort, the majority of published differences could not be reproduced, neither in our own nor in other NAFLD cohort studies.

Conclusion: Despite repeatedly reproduced abundance patterns of specific bacteria, the heterogeneous study results did not reveal a consistent disease specific gut microbiota signature. Further prospective studies with homogenous patient cohorts and standardized methods are necessary to phenotype NAFLD by the gut microbiota.
\end{abstract}

\section{Introduction}

Non-alcoholic fatty liver disease (NAFLD) is the most common chronic liver disease worldwide. ${ }^{1}$ It currently affects $20-30 \%$ of the general population with increasing prevalence. ${ }^{2}$ NAFLD encompasses a spectrum from simple steatosis to non-alcoholic steatohepatitis (NASH) with or without fibrosis to liver cirrhosis and hepatocellular carcinoma. ${ }^{3,4}$ NAFLD is strongly associated with features of the metabolic syndrome; insulin resistance and dyslipidemia represent major risk factors for hepatic fat and fibrosis development in NAFLD. ${ }^{5-7}$ There is rising evidence that alterations in the gut-liver axis are further involved in NAFLD pathogenesis. ${ }^{8}$ Intestinal dysbiosis can lead to increased in gut permeability ${ }^{9}$ intestinal endotoxemia, ${ }^{10}$ endogenous alcohol production, ${ }^{11}$ hepatic insulin resistance, ${ }^{12}$ and disruption of choline metabolism, ${ }^{13}$ which eventually might contribute to disease onset and progression. Several studies show alterations in the gut microbiota composition of NAFLD patients; however, results are inconsistent. Different analytical methods and study populations might contribute to this inconsistency; culture-based and culture-free methods like 16S rRNA gene sequencing as well as whole genome shotgun sequencing were used most widely. ${ }^{2,11,14-24}$

So far, a diligent comparison and validation of published findings from microbiota studies in NAFLD patients is missing. Therefore, the aim of this study was (i) to examine the 
reproducibility of previously published human gut microbiota data and (ii) to identify a consistent bacterial microbiota signature in patients associated with NAFLD and its disease severity. For this purpose, we analyzed preliminary results from our NAFLD cohort focusing exclusively on the question of replicability of already published data.

\section{Methods}

Study subjects. This cross-sectional, prospective study was designed to detect associations between the gut microbiota of patients with NAFLD and disease severity. The protocol was approved by the local ethics committee and written informed consent was obtained from all participants. NAFLD patients were recruited during regular follow-up visits in the outpatient liver department of the Clinic for Gastroenterology and Hepatology at the University Hospital of Cologne between March 2015 and December 2018. In addition, 21 healthy controls were additionally enrolled. NAFLD was diagnosed, if the following conditions were present: (i) hepatic steatosis on liver imaging and/or $>5 \%$ of hepatocytes storing fat on histological analysis of a liver biopsy and (ii) daily alcohol consumption of less than $10 \mathrm{~g}$ in women and less than $20 \mathrm{~g}$ in men, (iii) no regular use of medication known to cause fatty liver, (iv) no other diseases causing secondary steatosis, and (v) no other chronic liver disease.

Inclusion criteria for the healthy controls were (i) no history of any chronic disease, (ii) body mass index (BMI) $<25 \mathrm{~kg} / \mathrm{m}^{2}$, (iii) daily alcohol consumption of less than $10 \mathrm{~g}$ in women and less than $20 \mathrm{~g}$ in men, (iv) abdominal ultrasound without abnormalities, and (v) all measured laboratory parameters within the reference ranges.

Exclusion criteria for all study subjects were oral or intravenous antibiotic treatment within the last 6 months prior to the study, known malignancy, pregnancy, and age $<18$ years. Any recommendations, diagnosis, or treatment suggestions for study participants did not differ from usual patient care. Thus, NAFLD patients received the same overall lifestyle recommendations as indicated in the current European guideline. ${ }^{25}$ Further exclusion criteria for NAFLD patients were ongoing successful lifestyle modifications defined as more than $5 \%$ loss of body weight within the last 3 months prior to enrollment or current or prior participation in an interventional NASH study.

The metabolic syndrome was defined following the International Diabetes Foundation criteria. ${ }^{26}$ Type 2 diabetes was defined as glycated hemoglobin $(\mathrm{HbAlc}) \geq 48 \mathrm{mmol} / \mathrm{mol} \mathrm{Hb}$ and/or fasting glucose $\geq 126 \mathrm{mg} / \mathrm{dL}$ and/or use of antidiabetic medications. Overweight was defined as BMI $\geq 25 \mathrm{~kg} / \mathrm{m}^{2}$. Arterial hypertension was defined as office blood pressure $\geq 140 / 90 \mathrm{mmHg}$ on at least two measurements during at least two occasions or antihypertensive drug treatment.

Abdominal ultrasound was performed in all patients and healthy controls. Blood samples for laboratory analyses were collected in the fasting state and processed according to standard laboratory procedures (Cobas C 702, Roche Diagnostics, Rotkreuz, Switzerland; Cobas E 801, Roche Diagnostics, Rotkreuz, Switzerland; and CAPILLARIS 2 system, SEBIA, Evry Cedex, France).
Liver biopsies. If liver biopsy was performed, biospecimens were evaluated by experienced liver pathologists who were blinded for all clinical and laboratory data. The NASH Clinical Research Network histological scoring system $^{27}$ was used to evaluate disease activity and severity, and the NAFLD activity score (NAS) was obtained for each biopsy. In our study cohort, definite NASH was diagnosed when NAS was $\geq 4$ points with at least 1 point in each component while NAS $\leq 3$ points defined NAFL. Fibrosis was staged according to Kleiner et al. ${ }^{27}$ Advanced fibrosis was defined as stage 3 (bridging fibrosis) or 4 (cirrhosis). Six patients were staged as NASH without determination of histological grading based on the following findings: liver imaging consistent with liver cirrhosis (e.g. nodular hepatic contour, changes in volume distribution indicating portal hypertension in the absence of portal vein thrombosis, secondary phenomena of portal hypertension such as splenomegaly, enlarged caudate lobe and left lobe lateral segment, and regenerative nodules) together with clinical and laboratory signs of portal hypertension/cirrhosis (e.g. low platelets, albumin and prothrombin time, and esophageal varices).

Gut bacterial sequencing. The stool samples were collected by the patients themselves in a Fecotainer ${ }^{\circledR}$, transferred to standard laboratory stool tubes, and brought to the clinic within $3 \mathrm{~h}$ using provided coolpacks. Fecal samples were stored at $-80^{\circ} \mathrm{C}$ until further analyses. Unless otherwise indicated, all kits were used in accordance with the manufacturer's protocol. The DNA was isolated using the RNeasy Power Microbiome Kit (Qiagen, Hilden, Germany). Seven of the nine variable bacterial 16S rRNA gene regions (pool 1: V2, V4, and V8; and pool 2: V3, V6/7, and V9) were amplified with the Ion $16 \mathrm{~S}$ Metagenomics Kit (Thermo Fisher Scienctific, Waltham, USA) utilizing two primer pools (An integrated research solution for bacterial identification using $16 \mathrm{~S}$ rRNA gene sequencing on the Ion PGM $^{\mathrm{TM}}$ System with Ion Reporter ${ }^{\mathrm{TM}}$ software; https:// www.thermofisher.com/content/dam/LifeTech/Documents/PDFs/ Ion-16S-Metagenomics-Kit-Software-Application-ote.pdf).

Amplicons were pooled and cleaned using the NucleoMag NGS Clean-up (Macherey-Nagel, Düren, Germany). The Qubit system was used to determine amplicon concentration, and the library was prepared with the Ion Plus Fragment Library Kit (Thermo Fisher Scienctific, Waltham, USA). For the template preparation, amplicon concentration was diluted to $30 \mathrm{ng} / \mathrm{mL}$. The Ion Chef Kit and the Ion Chef system (both, Thermo Fisher Scienctific, Waltham, USA) were used to enrich and prepare the template-positive ion sphere particles. Metagenome was sequenced using the Ion Torrent $\mathrm{S} 5$ system ( $\mathrm{pH}$ dependent, Thermo Fisher Scienctific, Waltham, USA). Single-end Ion Torrent sequencing reads were analyzed with the proprietary Ion Reporter (version 5.10.5.0) platform using the Metagenomics $16 \mathrm{~S}$ w1.1 workflow. Briefly, unique sequences of at least 150 nucleotides length, and at least 10 read counts were aligned to the composite Curated MicroSEQ ${ }^{\circledR}$ 16S Reference Library (version 2013.1) and Curated GreenGenes (version 13.5) database requiring 90\% sequence coverage; taxonomy assignment required $97 \%$ sequence identity for genus level and 99\% identity for species level. 
Sequencing data have been deposited at National Center for Biotechnology Information sequence read archive in BioBioject PRJNA540738.

The raw data were processed with the programming language $\mathrm{R}$ version 3.5.1 using the $\mathrm{R}$ package phyloseq version 1.28.0. The feature table in biom format was imported using the function import_biom, and the resulting phyloseq-object was merged with patient (sample) data using the merge_phyloseq function. Alpha diversity was calculated using the estimate_richness function, which calls the functions estimateR and diversity from the vegan package (version 2.5-6) to calculate observed operational taxonomic units and Shannon Index, respectively (see code snippet in the Supporting Information).

Literature search. In order to identify relevant human studies investigating the bacterial gut microbiota composition in patients with NAFLD, PubMed, Google Scholar, and Scopus were searched using the terms "non-alcoholic or nonalcoholic fatty liver disease," "non-alcoholic or nonalcoholic steatohepatitis," "dysbiosis," "gut microbiome," and "gut microbiota" published prior to March 31, 2019. Only studies without intervention were included.
Subgroups. Study subjects were categorized according to the respective subgroups from the identified studies: "NAFLD with liver biopsy vs. healthy controls (HC)," "NAFLD without liver biopsy vs. HC," "NAFLD with liver biopsy + Obese vs. HC," "Nonobese NAFLD without liver biopsy vs. HC," "NAFL vs. HC," "NASH vs. HC," "NASH vs. NAFL," "NASH vs. NAFL + HC," "NASH + Obese vs. HC," "NAFLD F3-F4 vs. NAFLD F0-F2," and "NAFLD F0-F1 vs. NAFLD F2-F4."

Statistical analysis. Values were expressed as medians with interquartile ranges, if not stated otherwise. The bacterial operational taxonomic units were normalized to get the proportional, relative abundance of each bacterial taxon in each patient. Taxa with a relative abundance of more than $1 \%$ in any group were included in the statistical analysis. Differences between two groups were compared using the Wilcoxon-Mann-Whitney test for continuous variables and $\chi^{2}$ tests for categorical variables. For the comparison of more than two groups, Kruskal-Wallis analysis of variance with Dunn's post-hoc test and Holm correction was used. ${ }^{28}$

Statistical analysis was performed using R statistical software (R version 3.5.1, 2018 the R Foundation for Statistical Computing). A $P$ value of equal or less than 0.05 was considered as statistically significant.

Table 1 Baseline characteristics of the study population.

\begin{tabular}{|c|c|c|c|c|c|c|}
\hline & $\mathrm{HC}$ & NAFLD & $P$ value & NAFL & NASH & $P$ value \\
\hline Total $n$ & 21 & 90 & & 20 & 47 & $\mathrm{n} / \mathrm{a}$ \\
\hline Age, years & $38(25.0)$ & $50.5(24.8)$ & 0.08 & $42(24.5)$ & $57(20.0)$ & 0.010 \\
\hline Female, $n(\%)$ & $16(76.2)$ & $38(42.2)$ & 0.01 & $7(35.0)$ & $22(46.8)$ & 0.021 \\
\hline Albumin [35-52 g/L] & $45(1.0)$ & $45(4.0)$ & 0.87 & $46(4.0)$ & $44(3.5)$ & 0.105 \\
\hline Aspartate aminotransferase $[<50 \mathrm{U} / \mathrm{L}]$ & $24.5(7.8)$ & $33.5(22.8)$ & 0.001 & $30.5(7.5)$ & $38(26.5)$ & $<0.001$ \\
\hline Alanine aminotransferase $[<50 \mathrm{U} / \mathrm{L}]$ & $13(11.5)$ & $50.5(46.5)$ & $<0.001$ & $42(26.8)$ & $55(42)$ & $<0.001$ \\
\hline Gamma-glutamyl-transferase $[<60 \mathrm{U} / \mathrm{L}]$ & $12.5(13.8)$ & $80(85.2)$ & $<0.001$ & $82.5(87.8)$ & $81(84.5)$ & $<0.001$ \\
\hline Alkaline phosphatase [40-130 U/L] & $61(14.0)$ & $72.5(29.0)$ & 0.04 & 75 (33.3) & $74(28.5)$ & 0.068 \\
\hline Total bilirubin $[<1.2 \mathrm{mg} / \mathrm{dL}]$ & $0.5(0.5)$ & $0.5(0.4)$ & 0.49 & $0.6(0.4)$ & $0.5(0.5)$ & 0.444 \\
\hline Ferritin $[30-400 \mu \mathrm{g} / \mathrm{L}]$ & $81(64.5)$ & 203.5 (196.3) & 0.001 & $144.5(239.8)$ & $236(132.5)$ & 0.004 \\
\hline Fasting glucose [74-109 mg/dL] & $75.5(24.0)$ & $96.5(22.0)$ & $<0.001$ & $93.5(10.8)$ & $102(32.5)$ & $<0.001$ \\
\hline $\mathrm{HbA} 1 \mathrm{c}$ [28-38 mmol/mol] & $30(2.0)$ & $35.8(7.5)$ & 0.04 & $33(4.0)$ & $37.5(7.8)$ & 0.001 \\
\hline Body mass index $\left[\mathrm{kg} / \mathrm{m}^{2}\right]$ & $20(1.3)$ & $29.9(6.4)$ & $<0.001$ & $28.5(5.7)$ & $31.2(6.5)$ & $<0.001$ \\
\hline Waist circumference [cm] & $85(0.5)$ & $106(18.8)$ & $<0.001$ & $100(20.8)$ & $109(17.8)$ & $<0.001$ \\
\hline Metabolic syndrome, $n$ (\%) [yes] & $0(0.0)$ & 30 (33.3) & $\mathrm{n} / \mathrm{a}$ & $2(10)$ & $21(44.7)$ & 0.003 \\
\hline Antidiabetics, $n(\%)$ & $0(0.0)$ & $16(17.6)$ & $\mathrm{n} / \mathrm{a}$ & $0(0.0)$ & $13(27.7)$ & 0.007 \\
\hline Antihypertensives, $n(\%)$ & $0(0.0)$ & $34(37.4)$ & $\mathrm{n} / \mathrm{a}$ & $3(14.3)$ & $24(51.1)$ & 0.004 \\
\hline Statins, $n(\%)$ & $0(0.0)$ & $16(17.6)$ & $\mathrm{n} / \mathrm{a}$ & $2(9.5)$ & $11(23.4)$ & 0.179 \\
\hline Proton pump inhibitors, $n(\%)$ & $0(0.0)$ & $14(15.4)$ & $\mathrm{n} / \mathrm{a}$ & $1(4.8)$ & $9(19.1)$ & 0.122 \\
\hline NAFLD activity score & $\mathrm{n} / \mathrm{a}$ & $4(2.0)$ & $\mathrm{n} / \mathrm{a}$ & $2.5(1.0)$ & $5(2.0)$ & $\mathrm{n} / \mathrm{a}$ \\
\hline NAFLD fibrosis score & $-2.7(1.1)$ & $-2.3(2.1)$ & 0.13 & $-3.1(1.7)$ & $-1.6(2.6)$ & 0.006 \\
\hline Observed OTUs & $105(24)$ & $108(28.5)$ & 0.62 & $108.5(14.8)$ & 107 (33.5) & 0.947 \\
\hline Shannon diversity index & $3.5(0.2)$ & $3.4(0.3)$ & 0.40 & $3.5(0.2)$ & $3.3(0.4)$ & 0.123 \\
\hline
\end{tabular}

Reference values of laboratory parameters are given in squared brackets []. Values are given as median with interquartile range (IQR) in round brackets () if not stated otherwise. Missing values of overall cohort ( $n=111$ ): albumin, $n=13$; aspartate aminotransferase, $n=13$; alanine aminotransferase, $n=13$; gamma-glutamyl-transferase, $n=13$; alkaline phosphatase, $n=14$; total bilirubin, $n=14$; ferritin, $n=14$; fasting glucose, $n=13$; HbA1c, $n=25$; body mass index, $n=14$; waist circumference $n=26$; NAS, $n=6$ of biopsy-proven NAFLD (these six patients had clinically diagnosed NASH cirrhosis); NAFLD fibrosis score, $n=15$; no other missing values present.

HC, healthy controls; NAFLD, non-alcoholic fatty liver disease; NAFL, non-alcoholic fatty liver; NASH, non-alcoholic steatohepatitis; n/a, not applicable; OTU, operational taxonomic unit. 


\section{Results}

Clinical characteristics of our study population. A total of 90 patients with NAFLD were included in this study. Median age was 50.5 (interquartile range, 24.8) years. Twenty patients were diagnosed with NAFL and 47 with NASH. Twenty-one healthy individuals without liver biopsy were included as controls. Table 1 summarizes the baseline characteristics of the study cohort. BMI, waist circumference, blood pressure, fasting plasma glucose concentrations, $\mathrm{HbA1c}$, liver function tests, and ferritin were significantly higher in NAFLD patients as compared with healthy controls (Table 1).

Analyzed studies. Thirteen cross-sectional studies analyzing the gut bacterial microbiota composition in humans with NAFLD were included. Most of these studies performed 16S rRNA gene sequencing, obtained by different sequencing technologies, namely, pyrosequencing $2,11,14,16,18,20,22$ and Illumina NGS sequencing. ${ }^{15,17,23,24}$ Two studies used whole genome shotgun sequencing $^{19}$ and quantitative polymerase chain reaction, ${ }^{21}$ respectively.

The analysis of the taxonomic microbiota profiles revealed that the presence of NAFLD was associated with changes at phylum, class, family and genus level, suggesting that NAFLD is associated with specific intestinal microbiota signatures. ${ }^{2,11,14-24}$ The included studies compared heterogenous NAFLD cohorts: biopsy-proven NAFLD versus healthy controls, ${ }^{2,23}$ NAFLD without liver biopsy versus healthy controls, ${ }^{14,16-18}$ biopsy proven NASH ( \pm obesity) versus healthy controls, ${ }^{2,11,15,20-22}$ NASH versus NAFL, ${ }^{2,20,21,24}$ F0-1 versus F2-4 fibrosis, ${ }^{2,24}$ and F0-2 versus F3-4 fibrosis. ${ }^{19}$ Finally, the inter-study comparisons might be complicated by the fact that different studies did not compare the same taxonomic levels of the specific bacteria and that two studies exclusively investigated the gut microbiota composition of children and adolescents. ${ }^{11,20}$

Table 2 and Figure 1 summarize the observed differences in bacterial gut microbiota composition that were confirmed in at least two included studies. Study results, which were not confirmed in at least one other study, are not listed in this table but are summarized in detail in Table S1. In biopsy-proven NAFLD versus healthy controls, a significantly lower abundance of Bacteroidetes at phylum level was found in five studies ${ }^{2,20,21,23}$ and of the Ruminococcaceae abundance at family level in three studies, ${ }^{2,23}$ both including our own. The last finding was also confirmed in studies diagnosing NAFLD without liver biopsy. ${ }^{14,17,18}$ Veillonellaceae (family level) and Dorea (genus level) were significantly increased in NAFLD patients without liver biopsy in our study as well as in two others. ${ }^{16,18}$ In these two studies, as well as in the studies of Jiang et al. ${ }^{17}$ and Da Silva et al. ${ }^{23}$ a significantly higher abundance of the Lactobacillaceae family was further observed. This finding could be confirmed in our cohort of biopsy-proven NAFLD patients. Subgroup analyses of different patient groups with or without obesity or across the spectrum of NAFLD revealed comparable changes in gut microbiota composition in only two studies (Table 2; Fig. 1). Interestingly, there were comparable results between children with biopsy-proven NAFLD by Del Chierico et $a l^{20}$ and our adult participants with NAFLD: At phylum level, both showed higher abundances of
Actinobacteria as well as Dorea (phylum Firmicutes) and Ruminococcus (phylum Firmicutes, family Ruminococcaceae) at the genus level. Again, a lower abundance of the phylum Bacteroidetes was observed.

In summary, the most consistent differences found in the bacterial gut microbiota composition of NAFLD patients including our own cohort were a decreased abundance of Bacteroidetes (phylum) and Ruminococcaceae (family) as well as an increased abundance of the Lactobacillaceae and Veillonellaceae (families) and the Dorea (genus) in $5 / 14,{ }^{2,20,21,23} 6 / 14,{ }^{2,14,17,18,23} 5 / 14,{ }^{16-18,23}$ $3 / 14,{ }^{16,18}$ and $4 / 14^{16,18,20}$ studies, respectively.

\section{Discussion}

Accumulating data have linked gut microbiota dysbiosis to the presence and the severity of NAFLD. In this analysis, we could show that differences in bacterial gut microbiota composition between NAFLD and healthy controls were found at all bacterial taxonomic levels. Most consistently, NAFLD patients were characterized by a decreased abundance of Bacteroidetes and Ruminococcaceae as well as by an increased abundance of Lactobacillaceae, Veillonellaceae, and Dorea.

In recent years, several studies in mouse models implicate a causal relationship between gut microbiota dysbiosis and the development of NAFLD. ${ }^{29}$ NAFLD even seems to be a transmissible disease, fecal microbiota transfer from hyperglycemic and hyperinsulinemic mice induced features of NAFLD in germ-free raised recipient mice. ${ }^{30}$ However, causal relationships between gut bacterial dysbiosis and NAFLD development and progression in humans with NAFLD is lacking. ${ }^{31}$ Results from observational studies investigating the gut microbiome in NAFLD are inconsistent. ${ }^{32}$ Overall, as a common denominator, a lower bacterial diversity and dysbiosis in patients with NAFLD compared with controls has been observed. ${ }^{2,11,14-18,20-23}$ The reduced bacterial diversity could be attributable to different dietary habbits, genetic, and enviromental factors. These in turn contribute to insulin resistance and increased free fatty acids, which can lead to features of the metabolic syndrome. ${ }^{33}$ However, limited data exist to define a fecal microbiome signature that is specific for NAFLD. A reduced abundance of Bacteroidetes has been observed in the gut microbiota of NAFLD patients ${ }^{2,3,20,21,23}$ as well as in obese patients without NAFLD. ${ }^{34-38}$ Thus, it does not seem to be a NAFLD-specific observation. In line with Mouzaki et al. ${ }^{21}$ we detected a significant lower abundance of Bacteroidetes in NASH compared with NAFL patients. The reduced number of Ruminococcaceae in the gut microbiota of NAFLD patients seems to be independent of the BMI, age, and other metabolic risk factors. ${ }^{23}$ Further, the increased relative abundances of the families Veillonellaceae and Lactobacillacea as well as the genera Dorea and Streptococcus seem to be specifically associated with NAFLD, as these changes were not detected in studies that enrolled obese patients only. However, the abundances of these taxa were not observed when comparing NASH with NAFL patients. ${ }^{2,11,15,20-24}$ Some studies suggest that alterations in the gut microbiota are associated with the progression from NAFL to NASH as well as to advanced fibrosis: Loomba et al. ${ }^{19}$ observed a decreased number of gram-positive Firmicutes and an increase of gram-negative Proteobacteria (including Escherichia coli) in patients with advanced NASH fibrosis and suggested a microbiota 


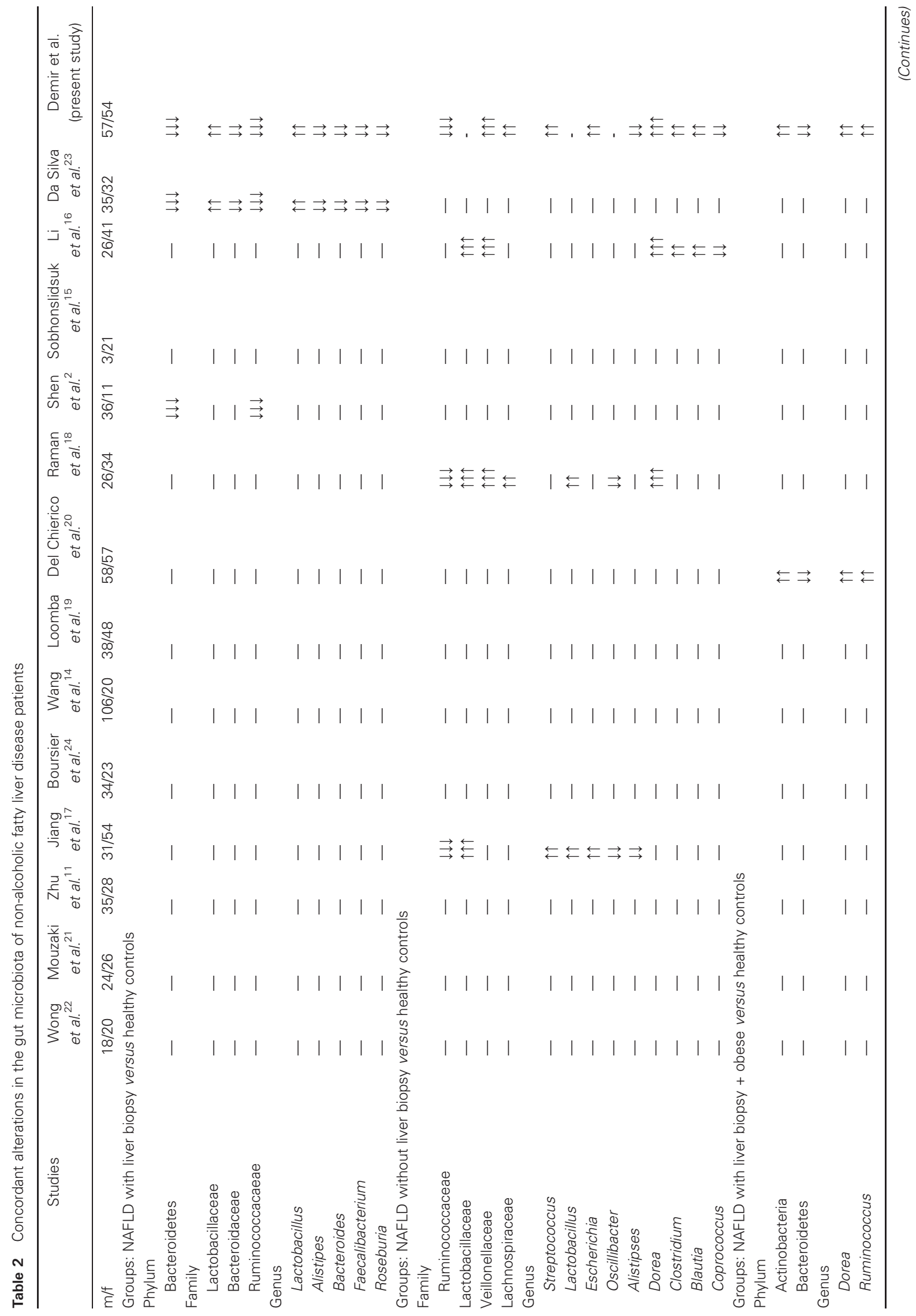




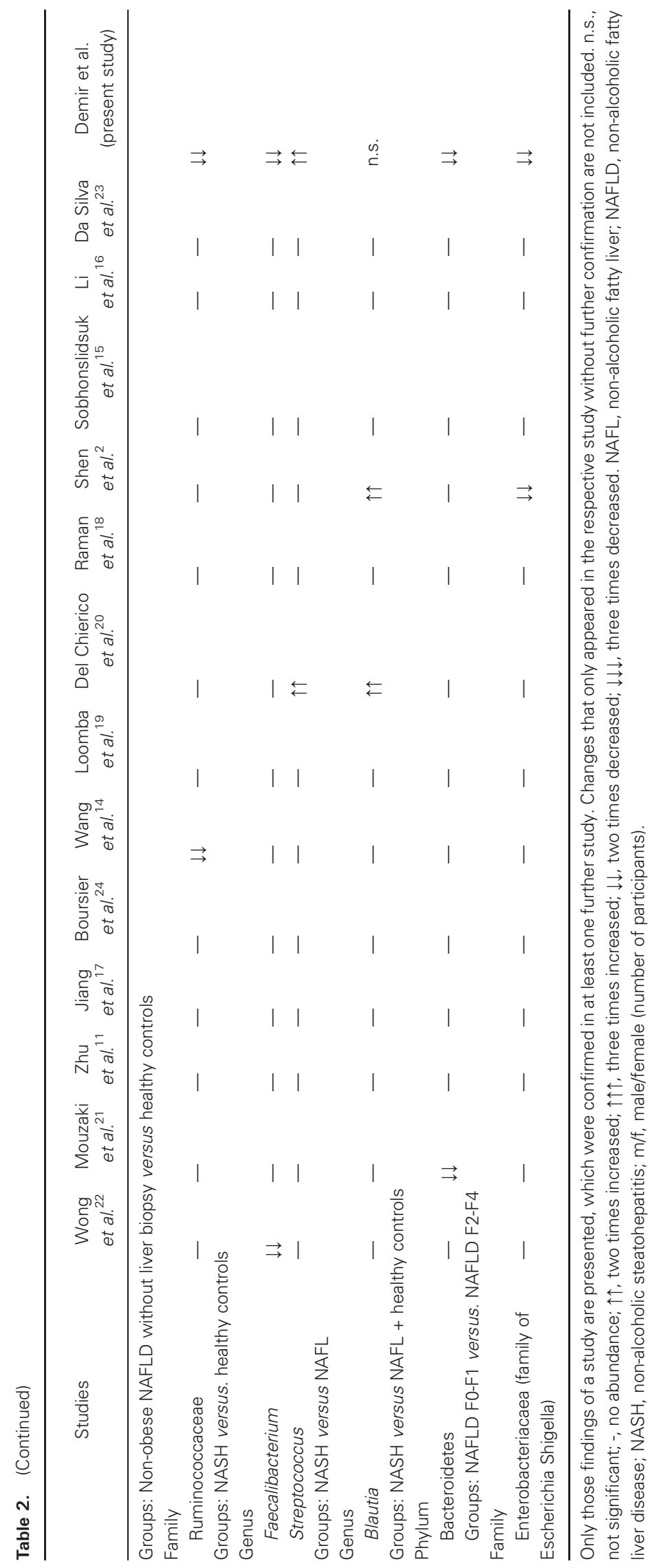



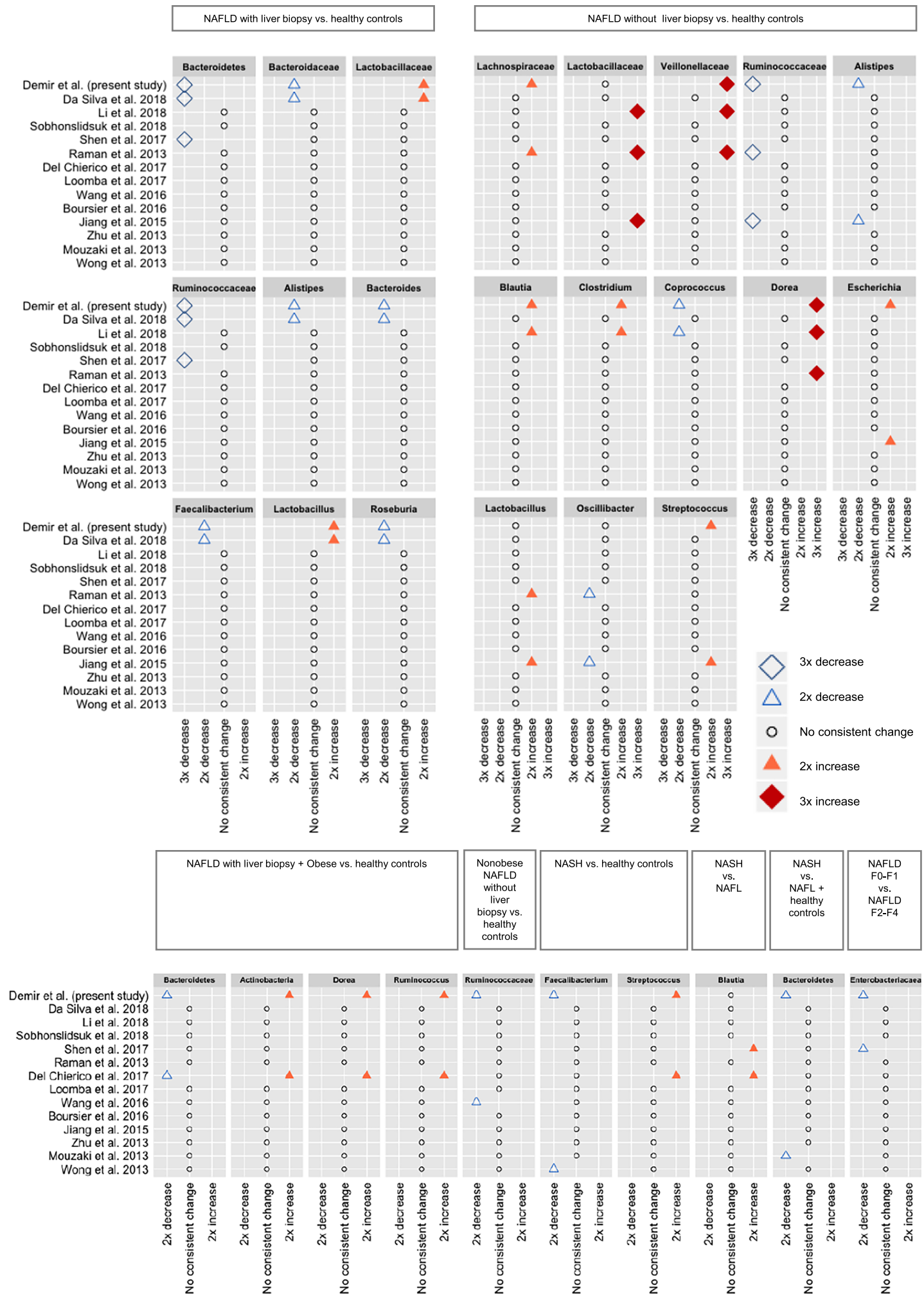

Figure 1 Summary of dysbiotic changes. Only those findings of a study are presented, which were confirmed in at least one further study. Reported changes that only appeared in the respective study without further confirmation are not included. [Color figure can be viewed at wileyonlinelibrary.com] 
shift towards a higher relative abundance of gram-negative bacteria. Eventually, these differences were used to non-invasisively detect advanced hepatic fibrosis in NAFLD.

Our study has several limitations. We compared studies that differ in patient populations, inclusion and exclusion criteria, as well as clinical characteristics, medication, and methods of microbiota analysis; adults and children were enrolled from various areas around the world with different environmental and nutritional conditions and different sequencing technologies were used. Because of these limitations, a detailed meta-analysis was not feasible. As detailed information on the mentioned points were often missing in the respective studies, corresponding controls for, for example, drugs like metformin or proton pump inhibitors or site-specific features could not be performed. Furthermore, we did not conduct metagenome analyses so we were not able to find out the potential consistency rules behind the observed inconsistencies (e.g. whether the inconsistent gut microbiota changes cause a consistent metabolic effect on the host). This is another limitation of our study. However, we would not have been able to compare the metagenome data as the majority of the included studies did not provide them either.

In summary, we provide a comprehensive comparison of previously published differences in the bacterial gut microbiota composition of NAFLD patients. A decreased abundance of the phylum Bacteroidetes and the family Ruminococcaceae as well as an increased abdundance of the families Veillonellacea and Lactobacillaceae and the genus Dorea were identified as the most consistent differences between NAFLD patients as compared with healthy controls. Even though these alterations could be reproduced in our cohort of NAFLD patients, the heterogeneous study results did not reveal a consistent gut microbiota signature in NAFLD. Further prospective studies in homogenous patient populations using standardized sequencing methods are needed to investigate a disease specific gut microbiota signature associated with NAFLD.

\section{Acknowledgments}

This study was supported by the "Marga und Walter BollStiftung," project 210-03-2016 and the "Köln Fortune" research pool, Faculty of Medicine, University of Cologne, Germany, project 160/2014 (to M. D.).

\section{References}

1 Younossi ZM, Koenig AB, Abdelatif D, Fazel Y, Henry L, Wymer M. Global epidemiology of nonalcoholic fatty liver disease-meta-analytic assessment of prevalence, incidence, and outcomes. Hepatology 2016; 64: $73-84$.

2 Shen F, Zheng RD, Sun XQ, Ding WJ, Wang XY, Fan JG. Gut microbiota dysbiosis in patients with non-alcoholic fatty liver disease. Hepatobiliary \& pancreatic diseases international: HBPD INT 2017; 16: $375-81$.

3 Singh S, Allen AM, Wang Z, Prokop LJ, Murad MH, Loomba R. Fibrosis progression in nonalcoholic fatty liver vs nonalcoholic steatohepatitis: a systematic review and meta-analysis of paired-biopsy studies. Clin Gastroenterol Hepatol 2015; 13: 643-54 e641-649; quiz e639-640.

4 Neuschwander-Tetri BA. Non-alcoholic fatty liver disease. BMC Med 2017; 15: 45.
5 Loomba R, Abraham M, Unalp A et al. Association between diabetes, family history of diabetes, and risk of nonalcoholic steatohepatitis and fibrosis. Hepatology 2012; 56: 943-51.

6 Yang KC, Hung HF, Lu CW, Chang HH, Lee LT, Huang KC. Association of non-alcoholic fatty liver disease with metabolic syndrome independently of central obesity and insulin resistance. Sci Rep 2016; 6: 27034.

7 Vanni E, Bugianesi E, Kotronen A, De Minicis S, Yki-Jarvinen H, Svegliati-Baroni G. From the metabolic syndrome to NAFLD or vice versa? Dig Liver Dis 2010; 42: 320-30.

8 Mehal WZ. The Gordian Knot of dysbiosis, obesity and NAFLD. Nat Rev Gastroenterol Hepatol 2013; 10: 637-44.

9 Miele L, Valenza V, La Torre G et al. Increased intestinal permeability and tight junction alterations in nonalcoholic fatty liver disease. Hepatology 2009; 49: 1877-87.

10 Harte AL, da Silva NF, Creely SJ et al. Elevated endotoxin levels in non-alcoholic fatty liver disease. J Inflamm (Lond) 2010; 7.

11 Zhu L, Baker SS, Gill C et al. Characterization of gut microbiomes in nonalcoholic steatohepatitis (NASH) patients: a connection between endogenous alcohol and NASH. Hepatology 2013; 57: 601-9.

12 Vijay-Kumar M, Aitken JD, Carvalho FA et al. Metabolic syndrome and altered gut microbiota in mice lacking toll-like receptor 5. Science 2010; 328: 228-31.

13 Dumas ME, Barton RH, Toye A et al. Metabolic profiling reveals a contribution of gut microbiota to fatty liver phenotype in insulin-resistant mice. Proc Natl Acad Sci U S A 2006; 103: 12511-6.

14 Wang B, Jiang X, Cao M et al. Altered fecal microbiota correlates with liver biochemistry in nonobese patients with non-alcoholic fatty liver disease. Sci Rep 2016; 6: 32002.

15 Sobhonslidsuk A, Chanprasertyothin S, Pongrujikorn T et al. The association of gut microbiota with nonalcoholic steatohepatitis in Thais. Biomed Res Int 2018; 2018: 9340316.

16 Li F, Sun G, Wang Z et al. Characteristics of fecal microbiota in non-alcoholic fatty liver disease patients. Sci China Life Sci 2018; 61: $770-8$.

17 Jiang W, Wu N, Wang X et al. Dysbiosis gut microbiota associated with inflammation and impaired mucosal immune function in intestine of humans with non-alcoholic fatty liver disease. Sci Rep 2015; 5: 8096.

18 Raman M, Ahmed I, Gillevet PM et al. Fecal microbiome and volatile organic compound metabolome in obese humans with nonalcoholic fatty liver disease. Clin Gastroenterol Hepatol 2013; 11: 868-75 e861863.

19 Loomba R, Seguritan V, Li W et al. Gut microbiome-based metagenomic signature for non-invasive detection of advanced fibrosis in human nonalcoholic fatty liver disease. Cell Metab 2017; 25: 1054$62 \mathrm{e} 1055$.

20 Del Chierico F, Nobili V, Vernocchi P et al. Gut microbiota profiling of pediatric nonalcoholic fatty liver disease and obese patients unveiled by an integrated meta-omics-based approach. Hepatology 2017; 65: 45164.

21 Mouzaki M, Comelli EM, Arendt BM et al. Intestinal microbiota in patients with nonalcoholic fatty liver disease. Hepatology 2013; 58: $120-7$.

22 Wong VW, Tse CH, Lam TT et al. Molecular characterization of the fecal microbiota in patients with nonalcoholic steatohepatitis-a longitudinal study. PLOS ONE 2013; 8: e62885.

23 Da Silva HE, Teterina A, Comelli EM et al. Nonalcoholic fatty liver disease is associated with dysbiosis independent of body mass index and insulin resistance. Sci Rep 2018; 8: 1466.

24 Boursier J, Mueller O, Barret M et al. The severity of nonalcoholic fatty liver disease is associated with gut dysbiosis and shift in the metabolic function of the gut microbiota. Hepatology 2016; 63: 76475. 
25 EASL-EASD-EASO clinical practice guidelines for the management of non-alcoholic fatty liver disease. J Hepatol 2016; 64: 1388-402.

26 Alberti KG, Zimmet P, Shaw J, Group IDFETFC. The metabolic syndrome - a new worldwide definition. Lancet 2005; 366: 1059-62.

27 Kleiner DE, Brunt EM, Van Natta M et al. Design and validation of a histological scoring system for nonalcoholic fatty liver disease. Hepatology 2005; 41: 1313-21.

28 Holm S. A simple sequentially rejective multiple test procedure. Scand J Stat 1979; 6: 65-70.

29 Schnabl B, Brenner DA. Interactions between the intestinal microbiome and liver diseases. Gastroenterology 2014; 146: 1513-24.

30 Le Roy T, Llopis M, Lepage P et al. Intestinal microbiota determines development of non-alcoholic fatty liver disease in mice. Gut 2013; 62: 1787-94.

31 Zmora N, Zilberman-Schapira G, Suez J et al. Personalized gut mucosal colonization resistance to empiric probiotics is associated with unique host and microbiome features. Cell 2018; 174: 1388-405 e1321.

32 Chu H, Williams B, Schnabl B. Gut microbiota, fatty liver disease, and hepatocellular carcinoma. Liver research 2018; 2: 43-51.

33 Leung C, Rivera L, Furness JB, Angus PW. The role of the gut microbiota in NAFLD. Nat Rev Gastroenterol Hepatol 2016; 13: 41225.

34 Furet JP, Kong LC, Tap J et al. Differential adaptation of human gut microbiota to bariatric surgery-induced weight loss: links with metabolic and low-grade inflammation markers. Diabetes 2010; 59: 3049-57.

35 Zhao L. The gut microbiota and obesity: from correlation to causality. Nat Rev Microbiol 2013; 11: 639-47.

36 Haro C, Rangel-Zuniga OA, Alcala-Diaz JF et al. Intestinal Microbiota Is Influenced by Gender and Body Mass Index. PLoS ONE 2016; 11: e0154090.

37 Ley RE, Turnbaugh PJ, Klein S, Gordon JI. Microbial ecology: human gut microbes associated with obesity. Nature 2006; 444: 1022-3.

38 Armougom F, Henry M, Vialettes B, Raccah D, Raoult D. Monitoring bacterial community of human gut microbiota reveals an increase in Lactobacillus in obese patients and Methanogens in anorexic patients. PLoS ONE 2009; 4: e7125.

\section{Supporting information}

Additional supporting information may be found online in the Supporting Information section at the end of the article.

Table S1. Overview of all alterations in gut bacterial taxa in NAFLD.

Data S1. Code snippet. 